\title{
Archéopages
}

Archéopages Archéologie et société

45 | 2017

De la terre au pot

\section{Les procédés de façonnage des potiers gaulois en}

\section{Cambrésis}

Analyse et reconstitution de céramiques

Gallic potters' shaping processes in Cambresis. Analysis and reconstitution of pottery

Los procedimientos de moldeado de los alfareros galos en Cambrésis. Análisis y reconstitución de cerámicas

David Bardel et Pierre-Alain Capt

\section{(2) OpenEdition}

\section{Journals}

Édition électronique

URL : https://journals.openedition.org/archeopages/2741

DOI : 10.4000/archeopages.2741

ISSN : 2269-9872

Éditeur

INRAP - Institut national de recherches archéologiques préventives

Édition imprimée

Date de publication : 1 mars 2018

Pagination : $32-41$

ISSN : 1622-8545

Référence électronique

David Bardel et Pierre-Alain Capt, «Les procédés de façonnage des potiers gaulois en Cambrésis », Archéopages [En ligne], 45 | 2017, mis en ligne le 01 janvier 2020, consulté le 02 juin 2021. URL : http:// journals.openedition.org/archeopages/2741 ; DOI : https://doi.org/10.4000/archeopages.2741 


\section{Les procédés de façonnage \\ des potiers gaulois en Cambrésis Analyse et reconstitutions expérimentales}

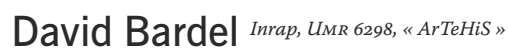

avec la collaboration de Pierre-Alain Capt archeoceramiste

\section{2}

1. Parc d'Activité Actipole de l'A2,

Raillencourt-Sainte-Olle

(Nord), RO J.-F. Geoffroy,

Inrap ; la Maladrerie-

CSNE-F2, Bourlon

(Pas-de-Calais),

RO D. Lamotte, Inrap ;

CSNE-F32, Marquion

(Pas-de-Calais),

RO J. Lamant, avec

P. Lefèvre, N. Sarrazin,

N. Soupart, Inrap.

2. Le corpus des vases

funéraires observés

regroupe un minimum

de 350 individus.

3. http://arscretariae

archeoceramique.

blogspot.fr

4. Collaboration autour

d'une reconstitution

du « vaisselier » gaulois

régional pour le parc

archéologique Asnapio

de Villeneuve-d'Ascq

(Nord).
Les fouilles archéologiques préventives conduites ces dernières années dans le Cambrésis, dans le cadre d'aménagements d'envergure - ZAC et canal à grand gabarit Seine-Nord-Europe ${ }^{1}$ (Geoffroy, en cours ; Lamotte et al., 2012 ; Prilaux et al., à paraître) -, ont mis au jour une documentation importante pour l'époque gauloise et particulièrement pour La Tène C et $\mathrm{D}$ ( 260 à 25 avant notre ère). Les collections céramiques, majoritairement funéraires, appartiennent à des dépôts en tombe à crémation. Ils offrent de nombreux vases complets ou semi-complets ${ }^{2}$ qui ont permis une caractérisation typologique et chronologique précise des répertoires (Bardel et al., 2016). Leur fragmentation importante du fait d'un écrasement sous le poids des terres, parfois lors de l'effondrement du coffrage d'une chambre funéraire, se prête à une analyse technologique plus systématique des procédés de façonnage.

Cet article aborde la caractérisation des traces et des procédés de montages observés pour ces différents corpus, en complémentarité avec quelques reconstitutions expérimentales réalisées par le potier « archéocéramiste » Pierre-Alain $\mathrm{Capt}^{\mathbf{3}}$, dans le cadre des activités du parc archéologique Asnapio ${ }^{\mathbf{4}}$ [encadrés 1 et 2]. L'objectif est d'évaluer les choix techniques de façonnage, les gestes et l'outillage des potiers afin de considérer les savoir-faire et les traditions des populations de Gaule Belgique, avant le développement du montage direct au tour qui n'intervient qu'à la fin de la période laténienne et au début de l'époque gallo-romaine.
La caractérisation des traces et l'identification des procédés de montage

Le protocole d'analyse est fondé sur l'observation des macrotraces caractéristiques des opérations de façonnage et de finition, visibles en surface des récipients et dans les tranches. Nous nous sommes appuyés pour cela sur les travaux de référence portant sur l'identification des traces et la reconnaissance des méthodes de façonnage, ainsi que sur une expérience d'identification initiée notamment dans le cadre des formations proposées par Valentine Roux. Si cette thématique de recherche s'est développée au cours des trente dernières années au travers d'études spécialisées - ethnographiques et archéologiques -, en association avec la mise en place de référentiels d'expérimentations (Rye, 1981; Balfet et al., 1983 ; Roux, 1994 ; Martineau, 2000 ; Livingstone Smith, 2001 ; Visseyrias, 2006 ; Gomart, 2014), ces travaux concernent surtout la période du Néolithique, voire l'âge du Bronze, et demeurent fort rares pour l'âge du Fer et la période laténienne (Augier, Renault, 2004; Durgeau, 2005 ; Desenne et al., 2005).

Les techniques de montage identifiées concernent le modelage, le moulage et le montage au colombin, parfois associé à un système de tournage (usage de l'énergie cinétique rotative, ECR). Les observations sont effectuées sur les trois grandes parties d'un vase - le fond, la panse et l'orifice - et concernent les deux grands stades de réalisation que sont l'ébauche et la finition. Les stigmates le plus fréquemment rencontrés consistent en la présence de ruptures linéaires préférentielles et la distinction de jointures dans la tranche, qui laissent penser que l'ébauche du vase est constituée de plusieurs éléments [ill. 1]. 
1. Exemples de macrotraces de façonnage par assemblage de colombins/bandes. Vase 1 (a): la vue de la tranche met en évidence la structuration interne et les zones d'assemblage de colombins (flèches rouges) qu'il convient de confronter à la vue interne du récipient (b) montrant les lignes horizontales de fractures. colombins.

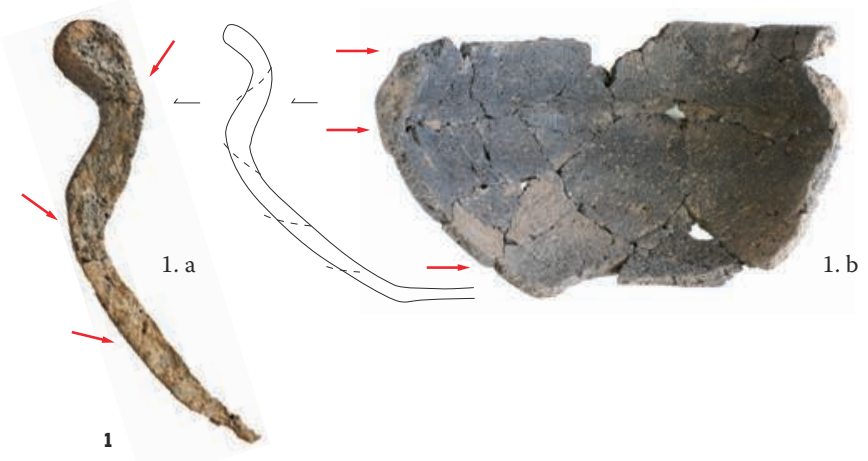

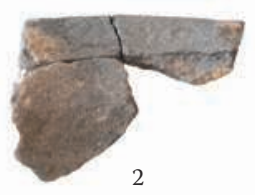

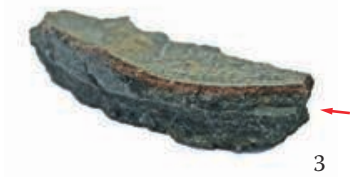

La production modelée sans usage du tournage

La production réalisée uniquement à main levée est le plus souvent majoritaire parmi les corpus analysés. Nous présentons ici les procédés de montage reconnus à partir de l'analyse des vases, que nous illustrons par des exemples caractéristiques.

\section{Le modelage de motte}

Cette technique consiste à creuser et étirer une motte d'argile. Elle se matérialise par des traces de pressions circulaires, d'étirements et par des différences d'épaisseur de paroi. L'homogénéité structurelle de la paroi et l'absence de cassures linéaires sont des indices supplémentaires. Ce procédé technique est peu utilisé pour le montage complet des récipients à l'exception de quelques microvases. Il est utilisé ponctuellement pour la réalisation de certaines galettes de fonds ou de toute la partie basse de récipients dont la partie haute est alors complétée selon la technique des colombins/bandes.

\section{Le montage aux colombins/bandes}

Cette technique d'assemblage qui consiste à superposer les colombins en anneau est la plus fréquente. Elle peut se remarquer en surface du vase par certains changements d'épaisseur, des dissymétries de volume et des traces irrégulières de régularisation des surfaces ou d'application du décor. Les observations des cassures mettent régulièrement en évidence des lignes horizontales qui témoignent de points de faiblesse ou de décollements au niveau des raccords des colombins/ bandes. On remarque également des changements d'inclinaisons et d'éventuels vides dans l'épaisseur de la tranche à la jonction de deux colombins.
Ces stigmates se rencontrent aussi bien sur des formes hautes que basses et, pour certains vases, ils permettent une lecture précise du nombre d'assemblages de bandes, de leur largeur et de leur sens de montage. Ce procédé est généralement utilisé pour le montage de l'ensemble du vase, mais permettrait également de compléter des ébauches de fond réalisées selon d'autres méthodes. La taille des colombins/bandes est variable selon les vases, en général 3 à $5 \mathrm{~cm}$, quelques fois plus larges.

Parmi les exemples sélectionnés au sein des corpus attribuables à La Tène $\mathrm{C}$ de Bourlon et de Marquion ou ceux de Raillencourt-Sainte-Olle, datés de La Tène $\mathrm{D}$, les formes basses à moyennes portent les traces d'assemblage de colombins d'épaisseur régulière [ill. 2]. Le vase $\mathrm{n}^{\circ} 1$ montre la réalisation d'une première galette d'argile centrale et l'adjonction d'une nouvelle bande pour former le large fond sur lequel viennent se rajouter, en trois étapes de façonnage, l'épaulement en ressaut, l'encolure et la lèvre épaissie. Pour les vases à fond plus étroit (exemples $n^{\circ} 2$ et 3 ), l'assise apparaît solidaire du bas de panse et les bandes sont rajoutées par des raccords obliques.

Les formes plus hautes et fermées impliquent les mêmes raccords obliques plus ou moins étirés, sur une assise qui semble généralement composée d'une seule pièce (exemple vase $n^{\circ} 4$ ), bien que dans certains cas elle soit faite de deux coupons d'argile (exemple vase 5).

\section{L'utilisation du moulage}

Le procédé de moulage peut être supposé pour la réalisation de la partie basse de certains individus. La technique peut consister en un moulage/étirement d'une plaque sur une forme concave ou convexe (céramique, récipient en bois, 
1

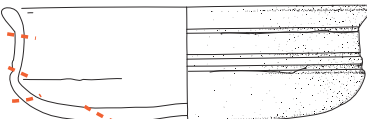

Bourlon CSNE F2 - 2010 - st. 104 n $^{\circ} 3$
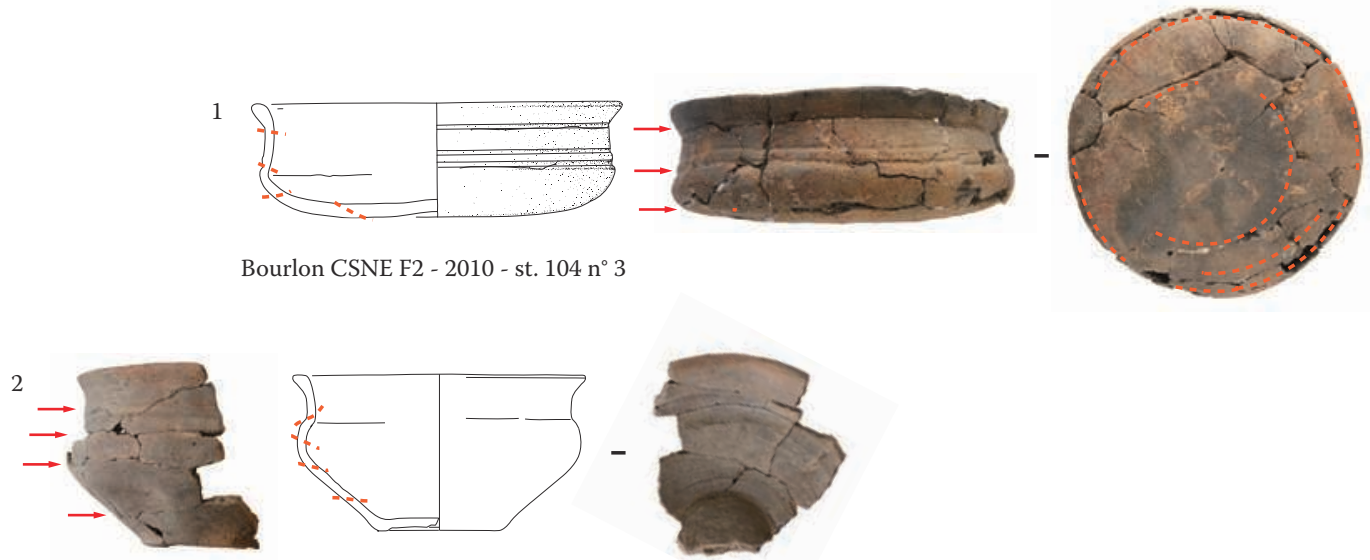

RSO-Actipole A2 - St. $2452 n^{\circ} 7$
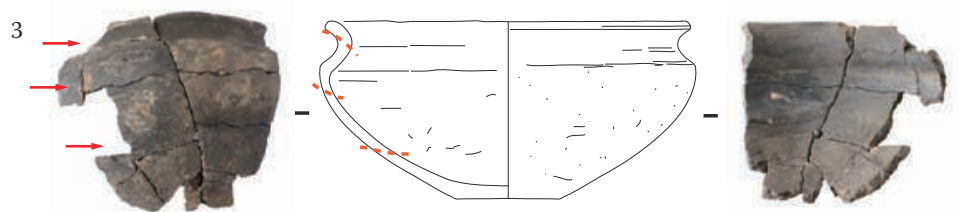

RSO-Actipole A2 - St. 1138 n $^{\circ} 2$

स

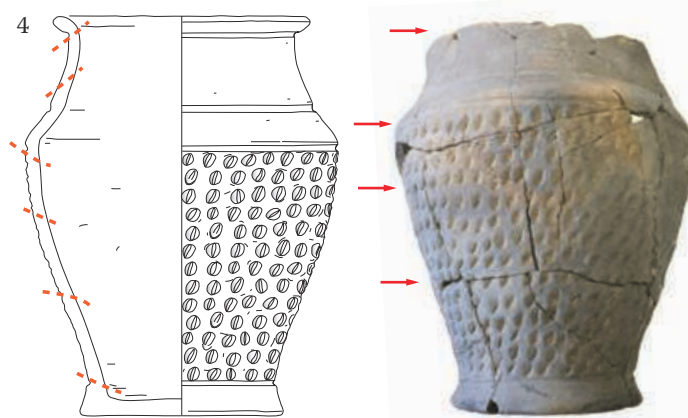

Marquion CSNE F 32-18 St. $332 \mathrm{n}^{\circ} 3$

$\rightleftharpoons \stackrel{\overline{c m}}{\rightleftharpoons} \rightleftharpoons$

vannerie...). Les traces diagnostiques correspondent à des différences d'épaisseur par zones, des marques de pressions ponctuelles plus ou moins importantes, des traces externes brutes aux aspects un peu émoussés ainsi qu'à l'absence de ligne de fracture horizontale. Dans l'épaisseur de la tranche ne se distinguent pas de jointures, mais de possibles zones d'orientations des particules en fonction des contraintes et des points de pression.

Ce procédé serait employé pour la réalisation de certaines parties basses de vases, dont les parties hautes relèveraient d'un complément par adjonction de bande/colombin : quelques exemples de jattes carénées des corpus de La Tène $\mathrm{C}$ attestent nettement de l'adjonction d'une encolure sur une base concave simple qui ne présente pas de traces de jointures, ni de ruptures linéaires [ill. $3, n^{\circ} 1$ et 2].

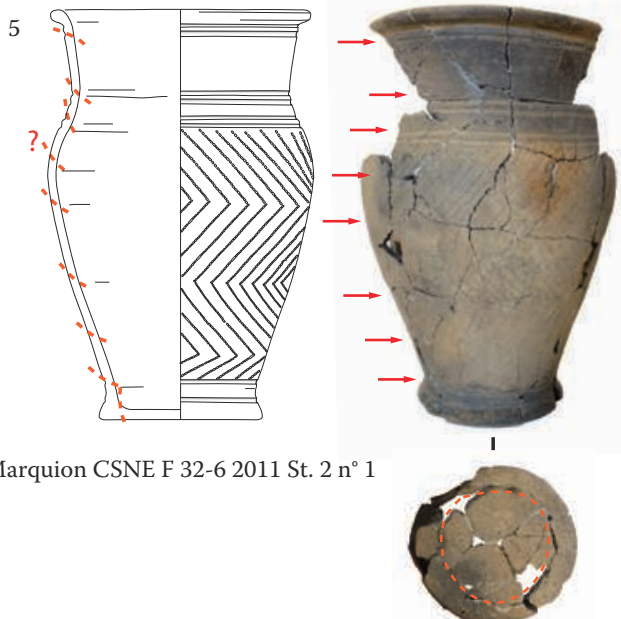

\section{La production modelée avec utilisation} de l'ECR

Les productions modelées faisant appel à un système de tournage sont nombreuses au sein des corpus funéraires. Si la régularité des surfaces et des décors est un critère important de la détermination, elle s'associe à leur morphologie sinueuse qui marque dès le début de La Tène moyenne une rupture avec les formes anguleuses de tradition ancienne (faciès caréné de l'AisneMarne). De plus, ces vases se distinguent généralement par une pâte fine sableuse spécifique et s'accompagnent de décors au lissoir et/ou de moulures. Une analyse des tranches et des ruptures linéaires permet d'identifier la réalisation d'une ébauche façonnée à la main par un assemblage de colombins, l'introduction d'un système de tournage se faisant soit lors de la mise 


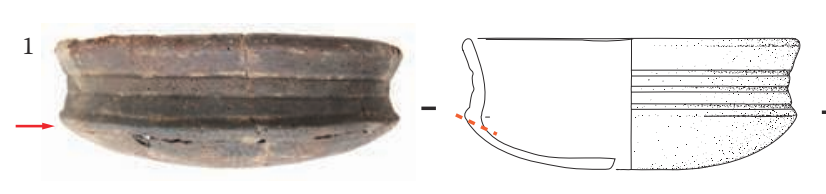

Bourlon CSNE F2 - 2010 - st. 73 n 4

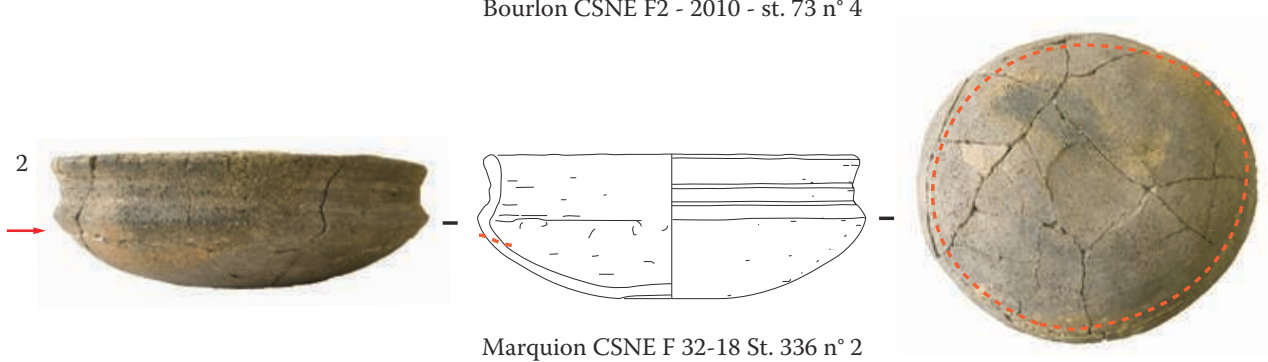

Marquion CSNE F 32-18 St. 336 n $^{\circ} 2$
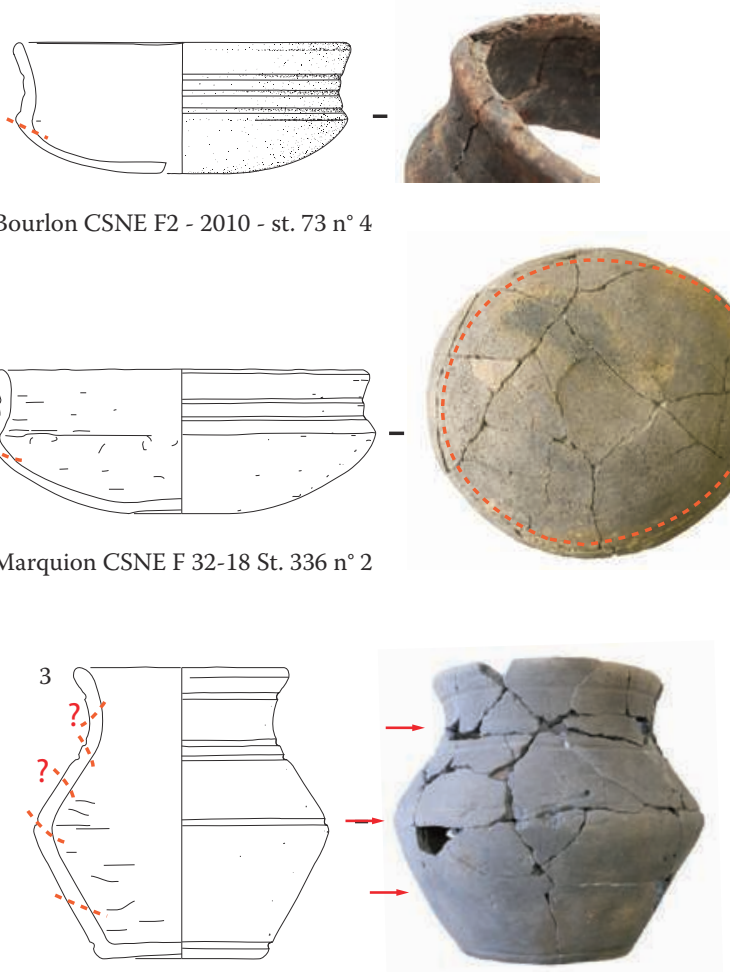

Marquion CSNE F 32-18 St. 353 n 4
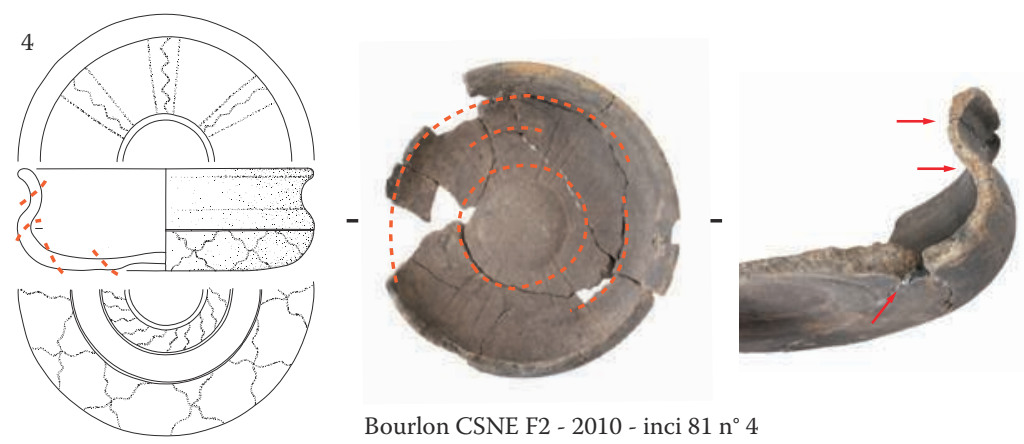

Bourlon CSNE F2 - 2010 - inci $81 n^{\circ} 4$ en forme des parois et du profil, soit lors de la finition.

\section{Le montage aux colombins/bandes avec l'utilisation du tournage en finition}

Un premier groupe est distingué par un usage de l'énergie cinétique rotative à un stade de finition, notamment pour la reprise des parties externes et de l'encolure.

On remarque la régularité des parois et des surfaces externes ainsi que la rectitude de décors cannelés, alors que les parties internes présentent, en plus ou moins grand nombre, des traces correspondant aux irrégularités du modelage et aux finitions à main levée. Différents degrés d'utilisation du tournage peuvent être perçus, impliquant certainement des habitudes et des dispositifs différents.
Des exemples de vases de La Tène $C$, de Marquion et de Bourlon, montrent une intervention de l'énergie cinétique rotative pour une régularisation en surface des parois externes et de l'encolure, ainsi que pour la réalisation du décor cannelé/incisé [ill. 3, $n^{\circ} 3$ et 4]. D'autres exemples de La Tène D provenant de Raillencourt-Sainte-Olle attestent d'une finition du vase à l'aide d'un système de tournage afin d'amincir et régulariser les parois ainsi que de réaliser le décor de baguettes ou cannelures [ill. $4, n^{\circ} 1$ et 2]. Le cas de la forme basse $n^{\circ} 1$, qui sert de modèle à la réalisation expérimentale de P.-A. Capt, illustre particulièrement cette étape dite de tournassage, permettant de reprendre, par raclage et creusement, le fond et les parois externes du vase pour la réalisation du décor, induisant ainsi une très faible épaisseur de la paroi centrale de l'anneau d'assise. Dans le cas de la 


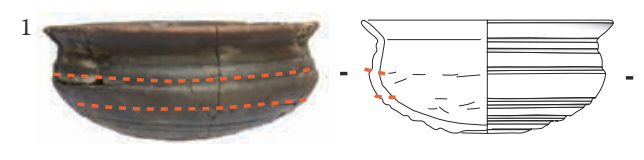

RSO-Actipole A2 - St. 516 n $^{\circ} 5$
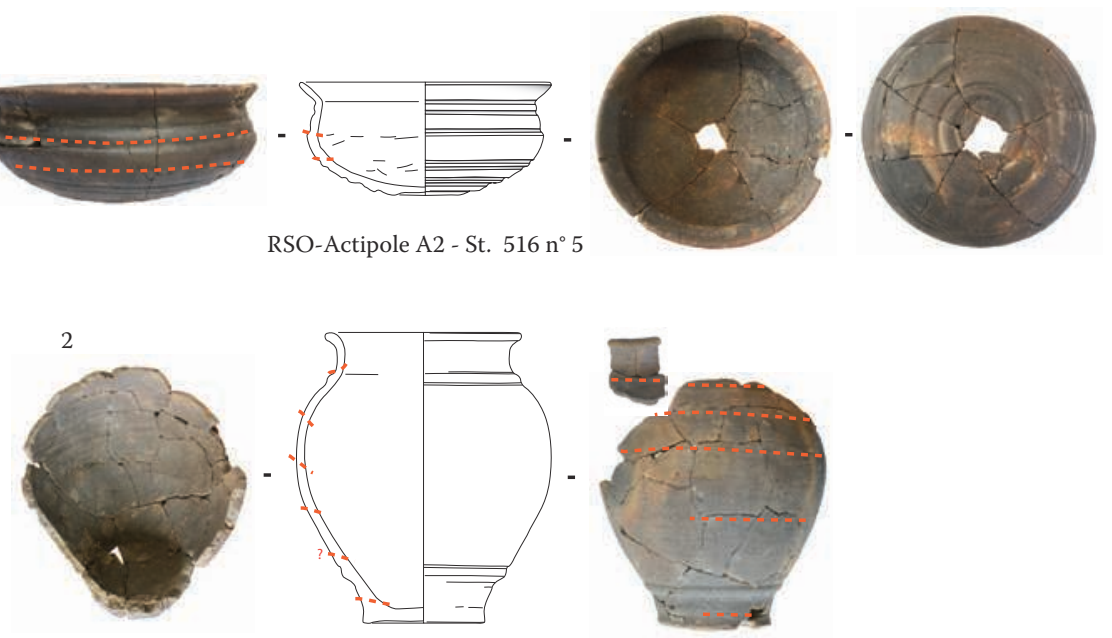

RSO-Actipole A2 - St. $2452 n^{\circ} 1$

3
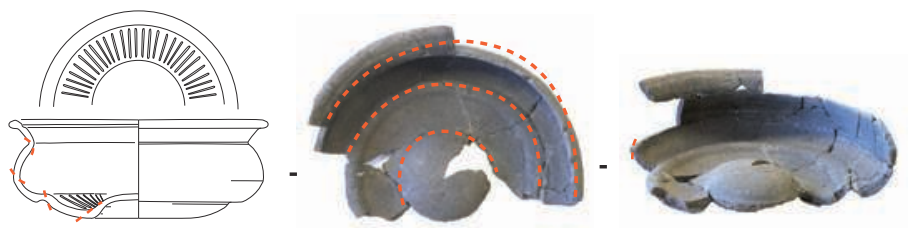

Marquion CSNE F 32-18 St. 345 n $^{\circ} 4$
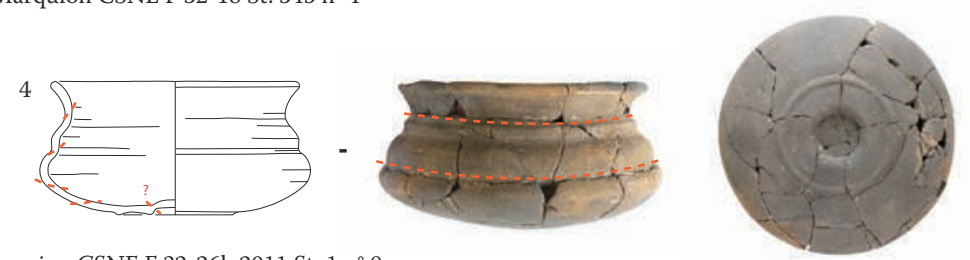

Marquion CSNE F 32-26b 2011 St. 1 n 8

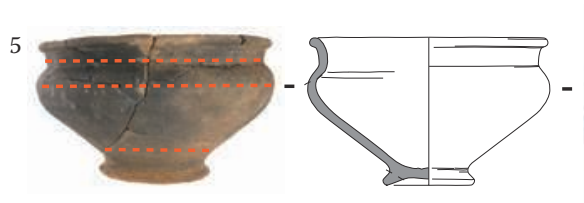

RSO-Actipole A2 - St. $2452 n^{\circ} 2$

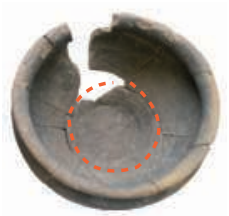

$\underset{0 \mathrm{~cm}}{\rightleftharpoons} \stackrel{=10}{\rightleftharpoons}$

bouteille $n^{\circ} 2$, l'ensemble de la panse externe et le décor de baguettes ont été façonnés sur la tournette par tournassage, alors que, pour les parties internes du vases, seuls l'épaulement et l'encolure présentent des traces de cet usage.

\section{Le montage aux colombins/bandes avec façonnage par tournage}

L'utilisation de l'énergie cinétique rotative pour la mise en forme du vase est assez fréquente pour la réalisation d'une vaisselle de table de belle qualité aux profils généralement curvilignes et décorée de moulures larges ou de cannelures. Les stigmates montrent une utilisation du tournage affectant toute l'épaisseur de la paroi : moulures larges, sillons horizontaux sur les parois internes et externes. Les jointures de colombins/bandes se manifestent toutefois par différentes lignes de fracture et sont également souvent visibles dans les tranches, certaines jonctions montrant un étirement et une superposition en biais importante, en lien avec à la reprise par tournage de l'ébauche déjà montée ou bien le façonnage par tournage de chaque bande rajoutée.

L'exemple d'une écuelle de Marquion datée de La Tène $C$ [ill. 4, n³] est évocateur des possibilités offertes par ce procédé mixte lorsqu'il est parfaitement maîtrisé : elle montre une mouluration importante de la partie basse munie d'un large omphalos et d'un anneau convexe. Ce façonnage de la paroi à l'aide de l'ECR, auquel pourrait s'ajouter une étape de tournassage, est également visible sur une autre écuelle à omphalos datant la fin de La Tène C [ill. 4, n 4], dont les parties hautes présentent de larges moulures. 

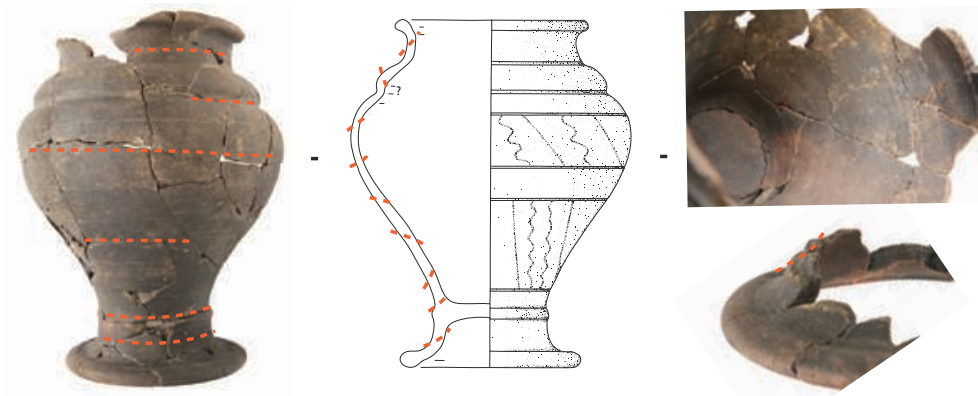

CSNE/Bourlon 2 - 2010 - inci $421 n^{\circ} 5$

2
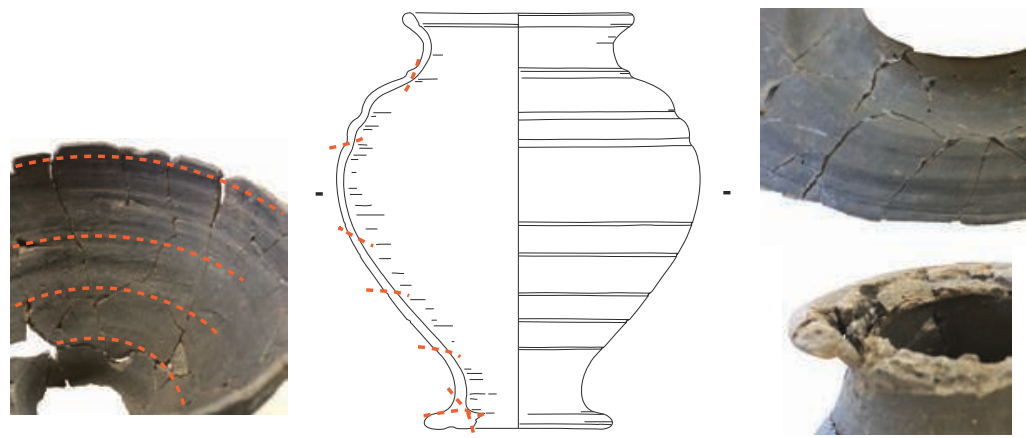

Marquion CSNE F 32-18 St. 335 n 5
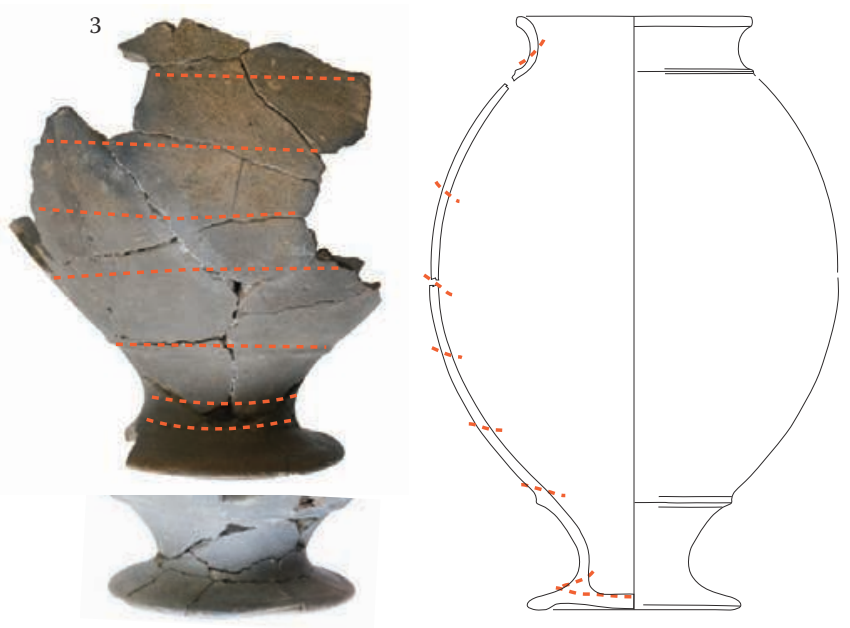

RSO Actipole A2 - St. $2452 n^{\circ} 6$
Certaines formes hautes de type bouteilles adoptent, dès le début de La Tène $C$, un profil novateur à épaulement arrondi et piédestal, représentatif d'une mise en forme à l'aide d'un système de tournage [ill. $5, n^{\circ} 1$ et 2]. La mouluration importante de l'épaulement et de l'encolure est à corréler avec des stigmates de façonnage par tournage très nettement visibles sur les parties internes (vase $\mathrm{n}^{\circ} 2$ notamment).

Les vases bouteilles de La Tène $\mathrm{D}$ évoluent vers des profils plus sinueux et élancés et adoptent des parois plus minces, à l'exemple de la bouteille à piédestal de Raillencourt-Sainte-Olle [ill. $5 \mathrm{n}^{\circ}$ 3]. Un assemblage par colombins/bandes se distingue clairement dans l'épaisseur des tranches. La chaîne opératoire de réalisation de ces vases évolue par rapport aux productions de La Tène $C$, puisque des traces de façonnage à main levée et quelques méplats sembleraient indiquer qu'une mise en forme de l'ébauche et un amincissement des parois aient été réalisés par battage et tournassage. La régularisation terminale de la paroi externe et la réalisation du décor de baguette attestent d'une finition par tournassage et d'un lustrage fin à l'aide du système rotatif.

\section{Les traditions potières et l'économie de la production céramique}

L'aperçu que nous dressons sur les aspects technologiques de montage s'appuie sur les analyses réalisées dans le cadre des post-fouilles. Une étude spécifique approfondie - observations complémentaires en lame mince et en radiographie notamment - permettrait une reconnaissance plus précise des chaînes opératoires. Cette analyse liminaire autorise néanmoins à identifier les 
principaux procédés de montage utilisés par les potiers du Cambrésis, complétant ainsi une approche culturelle et sociale de la production céramique et de son organisation.

L'analyse de ces corpus met en évidence des savoir-faire techniques relativement standardisés qui s'inscrivent dans des traditions anciennes, notamment pour ce qui concerne les montages par assemblage de colombins/bandes qui caractérisent une large période de la protohistoire régionale. L'utilisation d'un système de tournage dans le cadre d'une réalisation mixte, pour le façonnage de l'ébauche et/ou la finition des vases, se développe à partir de La Tène $C$ en même temps qu'un renouveau stylistique, pour la réalisation d'une vaisselle de table de qualité, souvent décorée au lissoir. Toutefois, ce procédé de montage doit aussi être perçu dans une certaine tradition technique entretenue depuis la fin du premier âge du Fer (Hallstatt D2/3), alors initiée au sein des complexes aristocratiques nord-alpins pour la réalisation d'un service de table prestigieux (Balzer, 2012 ; Bardel, 2012 ; Augier et al., 2013).

L'utilisation de dispositifs de tournage se poursuit tout au long de La Tène $\mathrm{D}$, accompagnant la réalisation de la vaisselle de table fine, mais également de formes plus courantes. Les procédés et les usages évoluent légèrement en même temps que les répertoires. Les parois des vases bouteilles s'amincissent et une régularisation des parties hautes ou des encolures par un tournassage devient plus fréquente, y compris pour les vases d'usage courant.

C'est avec la romanisation, dans le courant de la deuxième moitié du $\mathrm{I}^{\mathrm{er}}$ siècle avant notre ère, que se développent les premières productions montées au tour de type terra nigra, qui ne marquent toutefois aucunement la fin des productions modelées (Willems, Dubois, 2013).

Les dispositifs de tournage utilisés par les potiers ne nous sont pas connus. Toutefois, l'analyse technologique permettrait de restituer l'usage d'un tour à main, dit « tournette », qui en fonction de sa confection permet une vitesse de rotation et une inertie assez importante, à l'exemple de celle utilisée lors des expérimentations. La maîtrise du tour à main nécessite une expérience importante et un apprentissage spécialisé, qui est toutefois moins difficile à acquérir que la technique du tournage. Si l'on envisage l'existence d'artisans potiers spécialisés et de petits ateliers à diffusion locale pour les productions de belle qualité notamment façonnées au tour, la part des productions plus communes doit s'inscrire dans le cadre domestique, en complément d'autres activités artisanales ou agricoles.

Cette analyse des traces de montage permet donc une première connaissance des procédés techniques et de leur évolution. Elle souligne, d'une part, l'importance d'un montage traditionnel au colombin, et, d'autre part, l'existence des procédés complémentaires, parfois mixtes, notamment le développement important des productions associant au montage au colombin un système de tournage. Des études complémentaires seraient à envisager afin de préciser la variation des chaînes opératoires au sein de chaque corpus et de comparer précisément les techniques et traditions à l'échelle locale et régionale. 
Références bibliographiques

Augier L., Renault I., 2004, « Introduction de la force cinétique rotative dans la chaîne opératoire des productions du dernier quart du $\mathrm{VI}^{\mathrm{e}}$ et du $\mathrm{V}^{\mathrm{e}}$ siècle avant J.-C. à Bourges (Cher) : découvertes archéologiques et expérimentations ", in FEUGÈre M., GÉROLD J.-C. (dir.), Le tournage, des origine à l'an Mil. Actes du colloque de Niederbronn, octobre 2003, Montagnac, éditions M. Mergoil, « Monographies Instrumentum » 27, p. 101-111.

Augier L., BAlzer I., BArdel D. et al., 2013, «La céramique façonnée au tour, témoin privilégié de la diffusion des techniques au Hallstatt D et à La Tène A/B1 », in Colin A., Verdin F. (éd.) L'âge du Fer en Aquitaine et sur ses marges. Mobilité des hommes, diffusion des idées, circulation des biens dans l'espace européen à l'âge du Fer, Actes du $35^{\mathrm{e}}$ Colloque International de l'AFEAF, Bordeaux 2-5 juin 2011, Bordeaux, suppl. Aquitania 30, p. 563-594.

Balfet H., Fauvet-berthelot M.-F., Monzon S. 1984, Lexique et typologie des poteries, Paris, CNRS, $148 \mathrm{p}$.

BALZER I., 2012, « Technological innovations in pottery. Examples from celtic "princely" sites (6th to 5th century B.C.) in continental Europe », in Gauss W., KLEBINDER-Gauss G., vON RüdEN C. (éd.),

The transmission of technical knowledge in the production of ancient mediterranean pottery, Proceedings of International Conference at the Austrian Archaeological Institute at Athens, 23rd-25rd november 2012, Vienne, Österreichisches Archäologisches Institut, «Sonderschriften Band » 54, p. 139-163.

BARDEL D., 2012, Société, économie et territoires à l'âge du Fer dans le Centre-Est de la France : analyse des corpus céramiques des habitats du Ha D-LT A (VII ${ }^{e} V^{e}$ av. J.-C.), Thèse de doctorat, sous la direction de J.-P. Guillaumet et P. Barral, université de Dijon, 3 vol., $14,20 \mathrm{p}$.

Bardel D., Morel A., Willems S., 2016, « Chronologie des faciès mobiliers du Cambrésis de La Tène moyenne au début de l'époque romaine », in Blancquaert G., Malrain F., (dir.), Évolution des sociétés gauloises du second âge du Fer, entre mutations internes et influences externes. Actes $d u 8^{e}$ colloque international de l'AFEAF - Amiens

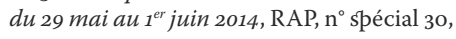
p. 495-520.

Desenne S., Collart J.-L., Auxiette G., Martin G., RAPIN A., 2005, «La nécropole d'Orainville "La Croyère" (Aisne). Un ensemble attribuable au Aisne-Marne IV ", Revue archéologique de Picardie, vol. 22, $\mathrm{n}^{\circ}$ 1, p. 233-29o.
Durgeau S., 2005, Mise en place de méthodes pour létude technique des céramiques de l'âge du Fer en Île-de-France, étude de cas : Le matériel du site "Le bâtiment hospitalier de l'Hôpital Avicenne » (Bobigny, Seine-Saint-Denis), Mémoire de DEA, université de Paris I, 135 p.

Geoffroy J.-F., en cours, Raillencourt-Sainte-Olle : extension du Parc d'Activités Actipole de l'A2, rapport d'opération, Inrap-SRA Hauts-de-France.

GOMART L., 2014, Traditions techniques et production céramique au Néolithique ancien : étude de huit sites rubanés du nord est de la France et de Belgique, Leiden, Sidestone Press, 342 p.

LAmotte D., BARdel D., Broes F. et al., 2012, Le site protohistorique et antique de La Maladrerie à Bourlon 2 (62) - Canal Seine Nord Europe, fouille 2, Pas-de-Calais, rapport d'opération, Inrap-SRA Nord-Pas-de-Calais, 535 p.

Livingstone SMith A., 2001, Chaînes opératoires de la poterie, référence ethnographiques, analyse et reconstitution, Thèse de doctorat, université de Bruxelles, $461 \mathrm{p}$.

Martineau R., 200o, Poterie, techniques et sociétés. Etudes analytiques et expérimentales à Chalain et Clairvaux (Jura), entre 3200 et 2900 av. J.-C., Thèse de doctorat, Besançon, université de Franche-Comté, $268 \mathrm{p}$.

Prilaux G., Soupart N., LefÈvre P. (dir.), à paraître, Du Néolithique à l'Antiquité tardive : les occupations de la plate-forme multimodale de Sauchy-LestréeMarquion (62), rapport d'opération, Inrap-SRA Hauts-de-France, $5 \mathrm{t}$

Roux V., 1994, « La technique du tournage définition et reconnaissance par les macrotraces », in Binder D., Courtin J., Terre cuite et société: la céramique, document technique, économique, culturel. Actes des $14^{\text {es }}$ Rencontres internationales d'archéologie et d'histoire (Antibes, 21-23 octobre 1993), Juan-les-Pins, APCDA, p. 45-58.

Rye O. S., 1981, Pottery technology. Principles and reconstruction, Washington, Taraxacum Press, $150 \mathrm{p}$.

VISSEYRIAS A., 2006, Les formes de la tradition: techniques et savoir-faire céramiques à la fin de l'âge $d u$ Bronze, entre Rhin et Rhône, Thèse de doctorat Besançon, université de Franche-Comté, 2 vol., $454 \mathrm{p}$

Willems S., Dubois S., 2013, « Le faciès céramique précoce des militaires et des civils, des vivants et des morts : données nouvelles en territoire nervien ", in Actes du congrès d'Amiens, 9-12 mai 2013, Marseille, SFECAG, p. 81-97. 

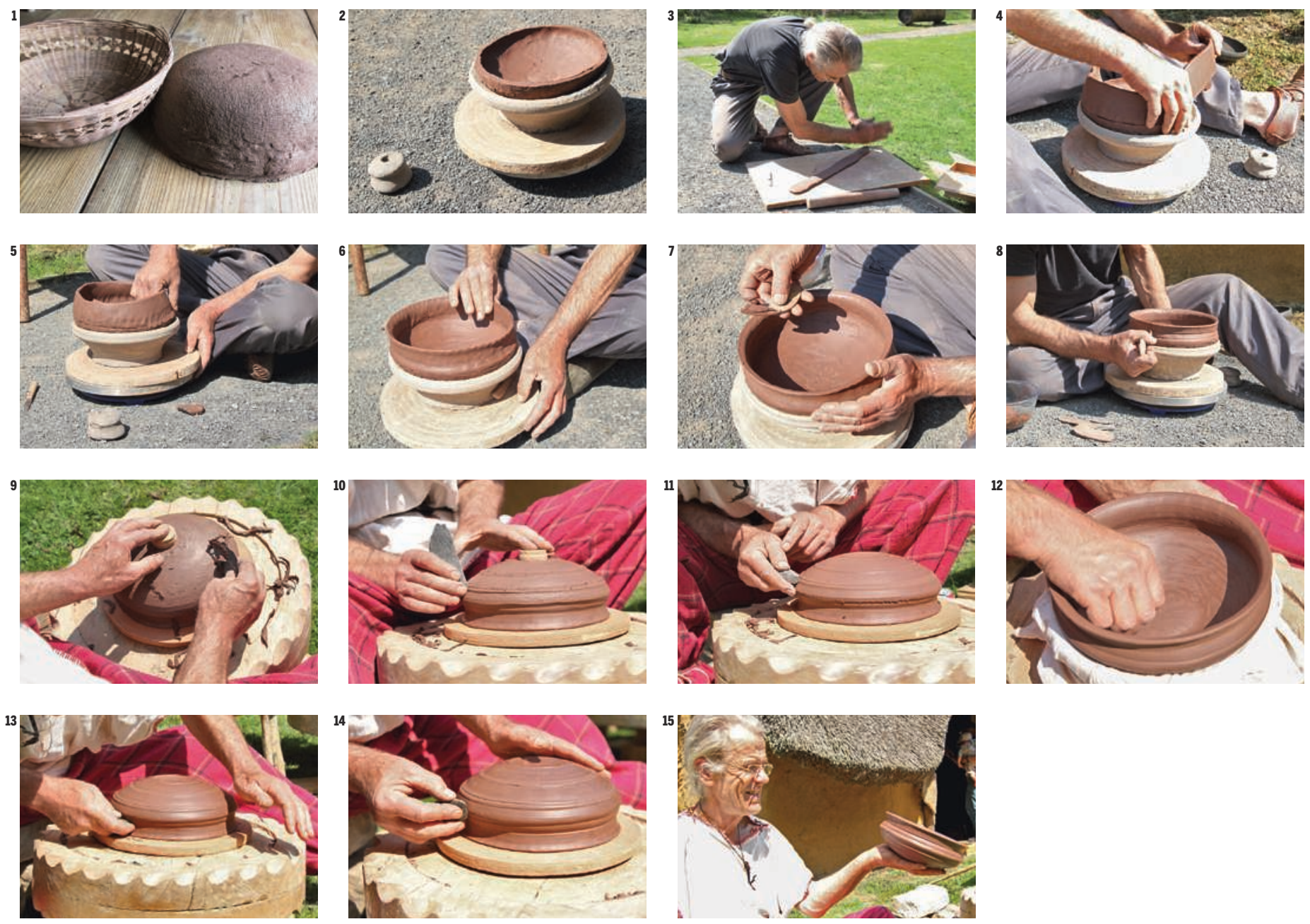

La reconstitution expérimentale : l'écuelle La reconstitution expérimentale d'une écuelle carénée est illustrée dans ses grandes étapes (réalisation par le potier P.-A. Capt) ; elle s'inspire d'un exemplaire du site de Raillencourt-Sainte-Olle daté de La Tène C2/D1 [ill. 4, $n^{\circ} 1$ ].

Partant du constat d'une certaine difficulté technique à réaliser des fonds ouverts et évasés à partir de colombins, ainsi que de l'absence de traces d'assemblage de colombins pour les parties basses de certains vases, le choix s'est porté vers une base réalisée par moulage d'une plaque d'argile à partir d'une vannerie (support qui pourrait également être d'une autre nature) (1). Une fois raffermie, cette base est démoulée puis martelée au sein d'une forme creuse en terre cuite à l'aide d'une masselotte (2). Entre-temps est façonnée une bande de terre (3), qui formera la partie haute (4). Elle est assemblée par pression et rabat sur l'ébauche de fond toujours maintenue dans sa forme creuse (5). En procédant par pincements et passages successifs, l'encolure est mise en forme, la régularité des gestes étant facilitée par la tournette (6). On procède ensuite, dès que le vase est suffisamment ferme pour être manipulé, à une première rectification de l'intérieur du vase, au moyen d'une estèque de bois (7). Une étape de rectification par tournassage est ensuite réalisée pour la partie externe du fond, de la panse et de l'encolure, en retournant le vase et en le posant sur le tour à main (8 et 9). Des plaquettes d'ardoise ou de bois permettent ce dégrossissage ; une réserve de matière est laissée pour les cordons / baguettes (9 et 10). Une étape de lissage/polissage au galet permet de régulariser l'aspect extérieur (11); un dernier polissage finalise la partie interne et laisse des traces similaires à celles observées sur la pièce archéologique (12). Enfin, une dernière reprise des surfaces externes au galet permet de finaliser le rendu du décor de baguettes et du poli de surface (13 et 14).

Cette possibilité technique nécessite un certain nombre d'opérations qui s'avèrent cependant relativement rapides et simples. Seules les étapes d'ébauchage et de rectification par tournassage demandent de l'expérience et une certaine formation technique, même si cette réalisation ne nécessite pas une maîtrise complète du métier de tourneur. 

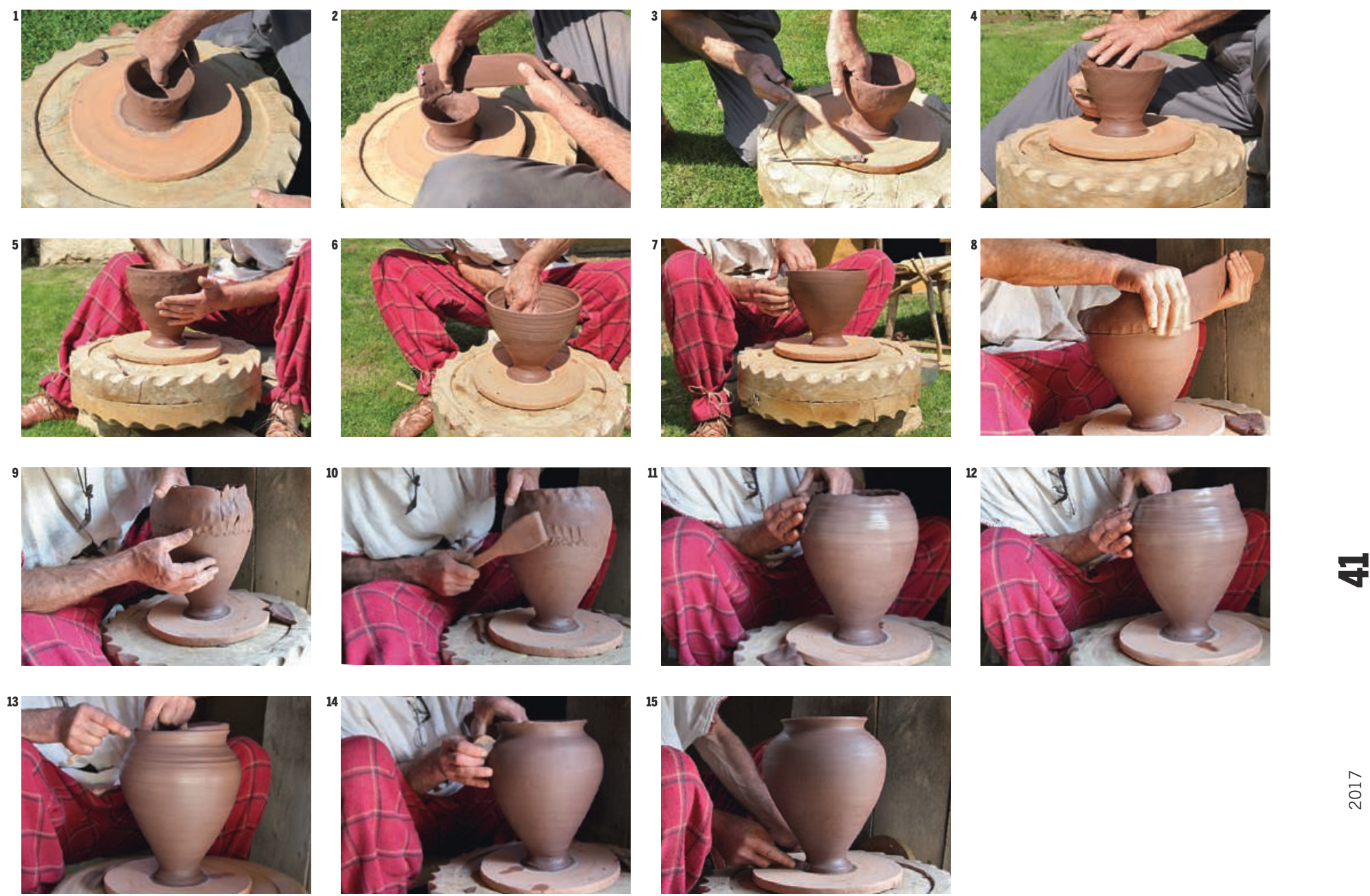

\section{La reconstitution expérimentale :}

\section{la bouteille}

Les traces observées sur plusieurs vases bouteilles du site de Raillencourt-Sainte-Olle permettent d'identifier une technique mixte de modelage par assemblage de colombin et tournage partiel (réalisation par le potier P.-A. Capt). Les surfaces externes de ces vases sont soigneusement lissées et les traces internes montrent les irrégularités de raclage et de façonnage, à l'exemple de la bouteille retrouvée dans une tombe $\left[i l l .5, n^{\circ} 3\right.$ ]. La restitution s'inspire de ces vases et s'appuie plus précisément sur un exemple non présenté, dont le fond n'a pas été rajouté en fin de montage.

Pour la reconstitution d'une telle pièce, le choix s'est porté vers un montage réalisé dès le début sur une tournette, mais qui pourrait également s'initier à main levée. Le pied du vase est modelé par creusage et expansion d'une balle d'argile que l'on fixe sur la tournette ( 1 ) et auquel on va rajouter une première bande assez large et aplatie, en la superposant sur la tranche. Le raccord est façonné de bas en haut sur la partie externe et en sens inverse pour la partie interne (2). On procède ensuite à une première régularisation et expansion des parois à l'aide d'un battu à la spatule /batte légère (3 et 4 ). On répète l'adjonction d'une nouvelle bande et son raccord soigneux (5). Puis un régularisation et un premier lissage par rotation peuvent être réalisés sur les parties externes et internes ( 6 et 7 ). Une dernière large bande est rajoutée par superposition; elle viendra former l'épaule et le col ( 8 et 9). Cette phase est la plus délicate, l'ensemble étant assez instable. Le haut de la panse est alors abondamment battu afin de lui donner sa forme finale et restreindre l'ouverture (10). Toutes les aspérités intérieures sont raclées et amincies à l'aide d'une estèque d'ardoise ou de bois. Une fois le bas de panse suffisamment raffermi, on peut commencer le tournage du haut du vase en étirant et en formant le col et la lèvre ( 11 et 12). Les irrégularités supérieures sont découpées, puis un lissage de la panse à l'ébauchoir et un premier polissage au galet sont réalisés ( 13 et 14). Après un léger séchage supplémentaire, les étapes de finitions au galet puis au chiffon ou à la peau de daim permettent d'obtenir le lustre final, en terminant par le pied qui peut alors être décollé de la tournette (15). 